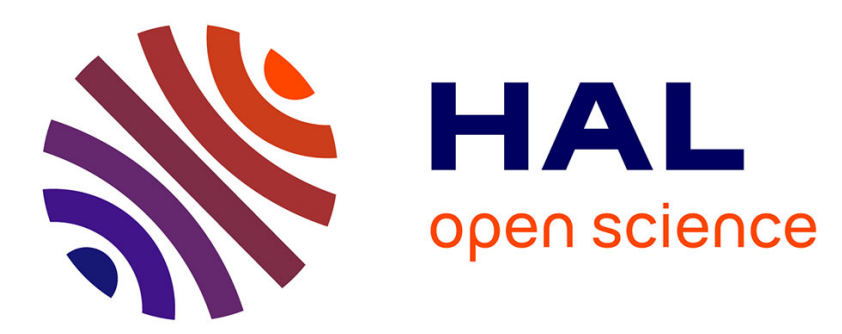

\title{
A multiscale computational approach with field transfer dedicated to coupled problems
}

\author{
David Dureisseix, David Néron
}

\section{To cite this version:}

David Dureisseix, David Néron. A multiscale computational approach with field transfer dedicated to coupled problems. International Journal for Multiscale Computational Engineering, 2008, 6 (3), pp.233-250. 10.1615/IntJMultCompEng.v6.i3.40 . hal-00330016

\section{HAL Id: hal-00330016 https://hal.science/hal-00330016}

Submitted on 1 Mar 2021

HAL is a multi-disciplinary open access archive for the deposit and dissemination of scientific research documents, whether they are published or not. The documents may come from teaching and research institutions in France or abroad, or from public or private research centers.
L'archive ouverte pluridisciplinaire HAL, est destinée au dépôt et à la diffusion de documents scientifiques de niveau recherche, publiés ou non, émanant des établissements d'enseignement et de recherche français ou étrangers, des laboratoires publics ou privés. 


\title{
A multiscale computational approach with field transfer dedicated to coupled problems
}

\author{
David Dureisseix ${ }^{\dagger, \star}$ and David Néron ${ }^{\ddagger}$ \\ ${ }^{\dagger}$ Laboratoire de Mécanique et Génie Civil (LMGC) \\ University Montpellier 2 / CNRS UMR 5508 \\ * Laboratoire de Micromécanique et d'Intégrité des Structures (MIST) \\ IRSN DPAM / CNRS UMR 5508 / University Montpellier 2 \\ CC 048, Place E. Bataillon, F-34095 MONTPELLIER CEDEX 5, FRANCE \\ David.Dureisseix@lmgc.univ-montp2.fr \\ $\ddagger$ Laboratoire de Mécanique et Technologie (LMT-Cachan) \\ ENS Cachan / CNRS UMR 8535 / University Paris 6 / PRES UniverSud Paris \\ 61, Avenue du Président Wilson, F-94235 CACHAN CEDEX, FRANCE \\ David.Neron@lmt.ens-cachan.fr
}

\begin{abstract}
This paper deals with a computational strategy suitable for the simulation of coupled problems, in presence of heterogeneities and when different precision levels are required for the different physics. To deal with micro heterogeneities, an adaptation of the classical periodic homogenization procedure is used, with the asymptotic development approach, but only one direction of periodicity can be taken into account. The application concerns an axisymmetric reinforced filtration device, modelled as a steady state thermo-poroelastic structure, for which thermal and fluid problems are described only at the (homogenized) macroscopic level, while the structure is described up to the micro scale. The relocalization has to take edge effects into account since scales are not well separated. The influence of the discretization on the micro scale is studied numerically.

This is a postprint of an article published in its final form as: David Dureisseix, David Néron, A multiscale computational approach with field transfer dedicated to coupled problems, International Journal for Multiscale Computational Engineering 6(3):233-250, Begell House, 2008. DOI: 10.1615/IntJMultCompEng.v6.i3.40. URL: Journal homepage.

Keywords: multiphysics; thermo-poroelasticity; periodic homogenization; partitioning; finite elements; non matching meshes; asymptotic expansion
\end{abstract}




\section{Introduction}

Multiphysics coupled problems often lead to different models or different discretizations. This may arise from the different engineering teams that focus on different phenomena, or because spatial (and temporal) scales may be different for each physics. In such cases, tools for coupling these models or discretizations are mandatory. They are classically associated to a partitioning technique as a solution strategy $[23,14,15,13,8]$, that allows modularity in the treatment of the different physics, and avoids extra numerical cost that can be associated to the coupling in a monolithic resolution.

In this article, we are concerned with the case where the spatial phenomenon scales of interest are different for the different physics. When heterogeneities are involved, the previous coupling tools must take into account the scale coupling as well. Multigrid methods often provide such scale coupling when coupled to the modelling phase $[2,16]$. The approach used herein is more related to domain decomposition [21].

To illustrate the topic of this article, let us consider the filtration device made of porous ceramic on Figure 1. To increase the flow rate, several design variables can be optimized: increasing the filtration surface by increasing inner diameter $D$ or length $2 L$, reducing the hydraulic resistance by diminishing thickness $e$, increasing the inflow pressure $p_{d}$. Each solution (except for the length increase that leads to an increase in axial bulk) leads to an increase in the orthoradial tensile stress $\sigma_{\theta \theta}$ for which the material does not exhibit a high resistance.

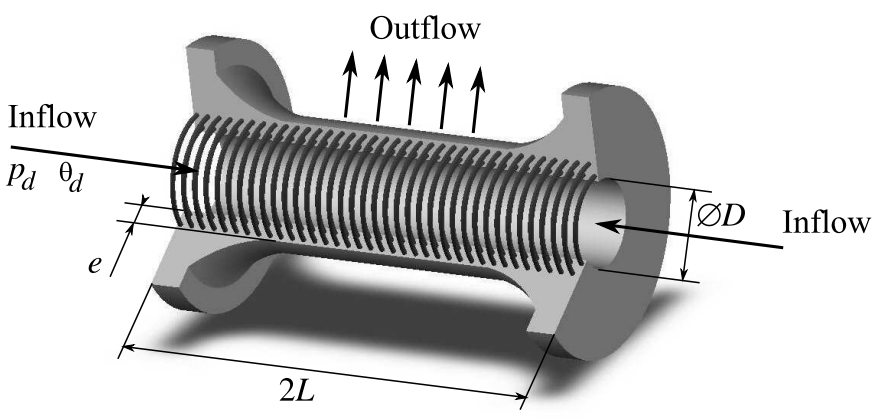

Figure 1: Filtration device

A design improvement in this case is to embed a reinforcement material when casting the device: for instance steel wires in the orthoradial direction as illustrated in Figure 1.

To check the integrity of such a structure, an axisymmetric thermo-poroelastic model can be used for the ceramic and a thermo-elastic model for the wires (the inflow temperature is $T_{0}+\theta_{d}, T_{0}$ being the reference temperature). Since the designer is mostly interested in the local stresses, and in the global flow rate that can be obtained, it will be of interest to describe the various physics with different finite element meshes: a fine mesh dedicated to capture local stresses for 
the elastic part, and a coarse mesh for both the fluid and the thermal problems. Such meshes are depicted in Figure 2 (half of the radial section is considered due to symmetry), and one can notice that the selected coarse mesh is unable to capture the effect of the reinforcement. Therefore, fluid and thermal problems have to use an homogenized macroscopic model, while the elastic problem may rely on the microscopic description of the 'composite' steel / ceramic structure.

(a)

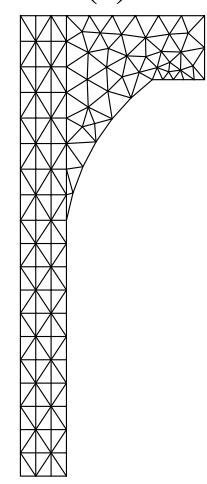

(b)

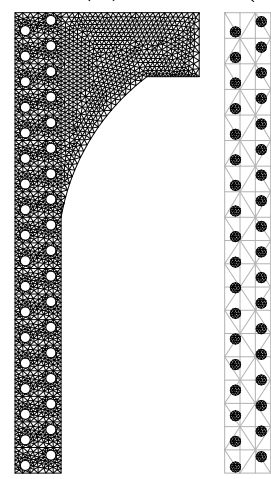

(c)

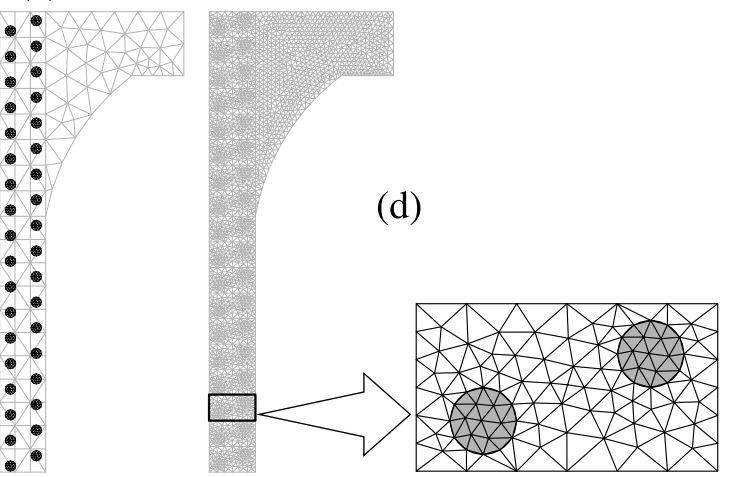

Figure 2: Finite element meshes of both the fluid and the thermal problems (a), of the porous solid (b), of the reinforcements (c), of the total solid problem and of a typical cell (d)

This article is therefore structured as follows: Section 2 will recall the reference problem; Section 3 will discuss the design of the homogenized model; Section 4 will introduce the field transfer procedure, based on the technique designed in $[12,25]$ and adapted herein to axisymmetric problems with partly homogenized models in a coupled physics problem. Finally, Section 5 will report numerical results and discussion on the model coupling procedure.

\section{Reference problem}

We recall herein the steady state governing equations of a saturated thermoporoelastic medium $\Omega[22,10]$. For a more complete presentation in the case of an evolution problem, the reader can refer to [25]. This kind of porous material model can be obtained from the underlying microstructure by homogenization [11]. Nevertheless, we won't consider this scale description herein. The micro scale will correspond only to the description of the composite structure made of a porous material and steel reinforcements; the macro scale will correspond to the homogenization of these two constituents.

When the temperature is $T_{0}+\theta$, with small variations from reference absolute temperature $T_{0}$, and with small perturbations, the state of the material is described at each point of the structure with the following parameters:

- for the solid part: the strain $\varepsilon$ associated to the stress $\sigma$; 
- for the fluid part: the pore pressure $p$ associated to the Darcy velocity $\underline{w}$;

- for the thermal part: the temperature $\theta$ associated to the generalized heat source $R$ and the thermal flux $\underline{q}_{\mathrm{th}}$.

Actually, in order to have positive material characteristics, we prefer to deal with $\underline{W}=-\underline{w}$, the opposite of Darcy velocity, and $\underline{Y}=-\underline{q}_{\mathrm{th}} / T_{0}$. The gradients involved are: $\underline{Z}=\operatorname{grad} p$, the gradient of the pore pressure, and $\underline{X}=\operatorname{grad} \theta$, the gradient of the temperature.

\subsection{Constitutive relations}

The constitutive relations are the following. First, the Hooke law is:

$$
\boldsymbol{\sigma}=\mathbf{D} \boldsymbol{\varepsilon}-b p \mathbf{I}-3 \alpha K_{b} \theta \mathbf{I}
$$

where $\mathbf{D}$ is the Hooke operator of the drained skeleton; for an isotropic behavior, it depends only on two coefficients, for instance, Young modulus $E$ and Poisson coefficient $\nu . \quad \alpha$ is the thermal (lineic) expansion coefficient of the skeleton. $K_{b}=\frac{1}{3} E /(1-2 \nu)$ is the bulk modulus of the drained skeleton. $b$ is Biot coefficient: $b=1-K_{b} / K_{s}$, where $K_{s}$ is the bulk modulus of the solid phase (solid grains).

Second, Darcy and Fourier laws read:

$$
\underline{W}=H \underline{Z} \quad \text { and } \quad \underline{Y}=\frac{k}{T_{0}} \underline{X}
$$

where $k$ is the thermal conductivity; as a first approximation, it can be chosen as $k=(1-n) k_{S}+n k_{F}, n$ being the porosity, $k_{S}$ and $k_{F}$ the thermal conductivities of the solid and fluid phases. $H=K / \mu_{F}$ is the permeability of the porous medium; $K$ is the intrinsic permeability of the skeleton, and $\mu_{F}$ is the dynamic viscosity of the fluid phase.

Finally, following [10], the thermodynamic framework leads to the thermal source:

$$
R=-\frac{\rho_{F} c_{F}}{T_{0}} \underline{W} \cdot \underline{X}-\frac{1}{H}\left(\frac{1}{T_{0}}-3 \alpha_{F}\right) \underline{W^{2}}
$$

where $\rho_{F}, c_{F}$ and $\alpha_{F}$ are the specific mass, the specific heat and the thermal expansion coefficient of the fluid phase. The two nonlinear terms in $R$ correspond to the heat convectively transported by the fluid, and to the viscous dissipation.

As an example of material characteristics of the problem that is under consideration, Tables 1 and 2 reports the parameter values. To avoid bad conditioning of the problem to solve, a new unit system is used, in order to have a unitary order of magnitude for $E, T_{0}, H$ and $C_{d} / T_{0}$ : lengths are in meters $(\mathrm{L}=\mathrm{m})$, masses in $710^{8} \mathrm{~kg}\left(\mathrm{M}=710^{8} \mathrm{~kg}\right)$, durations in seconds $(\mathrm{S}=\mathrm{s})$ and temperatures in $293 \mathrm{~K}(\mathrm{~T}=293 \mathrm{~K})$. Table 1 also gives the values in this new unit system.

The loadings on medium $\Omega$ consist of: 


\begin{tabular}{ll}
\hline Young modulus of the skeleton & $E=20 \mathrm{GPa}=28.57 \mathrm{M} \mathrm{L}^{-1} \mathrm{~S}^{-2}$ \\
Poisson coefficient of the skeleton & $\nu=0.2$ \\
Bulk modulus of the skeleton & $K_{b}=11.11 \mathrm{GPa}=15.87 \mathrm{M} \mathrm{L}^{-1} \mathrm{~S}^{-2}$ \\
\hline Biot coefficient & $b=0.7$ \\
Porosity & $n=0.2$ \\
Intrinsic perm. of the skeleton & $K=210^{-13} \mathrm{~s} \mathrm{~m}^{2}=210^{-13} \mathrm{~S} \mathrm{~L}^{2}$ \\
Dynamic viscosity of the fluid & $\mu_{F}=0.001 \mathrm{~Pa} \mathrm{~s}^{-1}=1.4310^{-12} \mathrm{M} \mathrm{L}^{-1} \mathrm{~S}^{-1}$ \\
Permeability of the porous media & $H=210^{-10} \mathrm{~m}^{3} \mathrm{~s} \mathrm{~kg}^{-1}=0.14 \mathrm{~L}^{3} \mathrm{~S} \mathrm{M}^{-1}$ \\
\hline Thermal conductivity of the solid & $k_{S}=85 \mathrm{~W} \mathrm{~m}^{-1} \mathrm{~K}^{-1}=3.5610^{-5} \mathrm{M} \mathrm{L} \mathrm{S}^{-3} \mathrm{~T}^{-1}$ \\
Thermal conductivity of the fluid & $k_{F}=0.61 \mathrm{~W} \mathrm{~m}^{-1} \mathrm{~K}^{-1}=2.5510^{-7} \mathrm{M} \mathrm{L} \mathrm{S}^{-3} \mathrm{~T}^{-1}$ \\
Thermal cond. of the porous media & $k=68 \mathrm{~W} \mathrm{~m} \mathrm{~K}^{-1}=2.8510^{-5} \mathrm{M} \mathrm{L} \mathrm{S}^{-3} \mathrm{~T}^{-1}$ \\
Specific mass of the fluid & $\rho_{F}=1000 \mathrm{~kg} \mathrm{~m}^{-3}=1.4310^{-6} \mathrm{M} \mathrm{L}^{-3}$ \\
Specific heat of the fluid & $c_{F}=4182 \mathrm{~J} \mathrm{~kg}^{-1} \mathrm{~K}^{-1}=1.2310^{6} \mathrm{~L}^{2} \mathrm{~S}^{-2} \mathrm{~T}^{-1}$ \\
Thermal exp. coef. of the skeleton & $\alpha=810^{-6} \mathrm{~K}^{-1}=2.3410^{-3} \mathrm{~T}^{-1}$ \\
Thermal exp. coef. of the fluid & $\alpha_{F}=2.610^{-4} \mathrm{~K}^{-1}=7.6210^{-2} \mathrm{~T}^{-1}$ \\
Reference temperature & $T_{0}=293 \mathrm{~K}=1 \mathrm{~T}$ \\
\hline
\end{tabular}

Table 1: Material characteristics (porous ceramics)

\begin{tabular}{ll}
\hline Young modulus & $E=200 \mathrm{GPa}=285.7 \mathrm{M} \mathrm{L}^{-1} \mathrm{~S}^{-2}$ \\
Poisson coefficient & $\nu=0.3$ \\
Bulk modulus & $K_{b}=167 \mathrm{GPa}=79.36 \mathrm{M} \mathrm{L}^{-1} \mathrm{~S}^{-2}$ \\
\hline Thermal conductivity & $k=20 \mathrm{~W} \mathrm{~m}^{-1} \mathrm{~K}^{-1}=8.4810^{-6} \mathrm{M} \mathrm{L} \mathrm{S}^{-3} \mathrm{~T}^{-1}$ \\
Thermal expansion coefficient & $\alpha=10.910^{-6} \mathrm{~K}^{-1}=3.210^{-3} \mathrm{~T}^{-1}$ \\
\hline
\end{tabular}

Table 2: Material characteristics (steel reinforcement) 
- prescribed displacement $\underline{U}_{d}$ on a first part $\partial_{1} \Omega$ of the boundary $\partial \Omega$ and traction force $\underline{F}_{d}$ on the complementary part $\partial_{2} \Omega$ of $\partial \Omega$;

- prescribed pore pressure $p_{d}$ on another part $\partial_{3} \Omega$ of the boundary and fluid flux $w_{d}$ on the complementary part $\partial_{4} \Omega$ of $\partial \Omega$;

- prescribed temperature $\theta_{d}$ on another part $\partial_{5} \Omega$ of the boundary and, finally, thermal flux $g_{d}$ on the complementary part $\partial_{6} \Omega$ of $\partial \Omega$.

For sake of simplicity, we assume that there are no body force, no fluid source and no thermal source, except $R$.

Once the constitutive relations are settled for the material behavior, the conservation principles have to be established: the momentum conservation for the solid, the mass conservation for the fluid, and the heat equation. These are the global admissibility relations.

\subsection{Solid admissibility}

Concerning the solid problem, one must have a compatible strain field $\varepsilon$ that equals the symmetric part of the gradient of a displacement field $\underline{U}$. This displacement field should be regular and equals the prescribed displacement $\underline{U}_{d}$ on a first part $\partial_{1} \Omega$ of the boundary $\partial \Omega$ of the domain (the corresponding set of fields $\underline{U}$ is denoted with $\mathcal{U}$ ). The stress field should also balance the external prescribed forces $\underline{F}_{d}$ on the complementary part $\partial_{2} \Omega$ of the domain and should also satisfy the momentum conservation. Therefore, one should have:

$$
\begin{array}{ll}
\varepsilon=\left(\frac{\partial \underline{U}}{\partial \underline{M}}\right)_{\text {sym }} \text { and } \quad \underline{U} \in \mathcal{U} \\
\underline{\operatorname{div}} \boldsymbol{\sigma}=\underline{0} \text { in } \Omega \quad \text { and } \quad \boldsymbol{\sigma} \underline{n}=\underline{F}_{d} \text { on } \partial_{2} \Omega
\end{array}
$$

\section{$2.3 \quad$ Fluid admissibility}

Concerning the fluid problem, the pressure gradient should derive from a regular pore pressure field that equals the prescribed values $p_{d}$ on another part of the boundary $\partial_{3} \Omega$ (the corresponding set of fields $p$ is denoted with $\mathcal{P}$ ). The fluid flux must equal the prescribed values on the complementary part $\partial_{4} \Omega$ of the boundary and satisfy conservation of fluid flow. Therefore, one should have:

$$
\begin{aligned}
& \underline{Z}=\underline{\operatorname{grad}} p \quad \text { and } \quad p \in \mathcal{P} \\
& \operatorname{div} \underline{W}=0 \text { in } \Omega \text { and } \underline{W} \cdot \underline{n}=w_{d} \text { on } \partial_{4} \Omega
\end{aligned}
$$

\subsection{Thermal admissibility}

Concerning the thermal problem, one must have a temperature gradient $\underline{X}$ arising from a regular temperature field $\theta$ that equals the prescribed value $\theta_{d}$ on another part $\partial_{5} \Omega$ of the boundary (the corresponding set of fields $\theta$ is denoted 
with $\mathcal{T}$ ). The thermal flux must equal the prescribed values on the complementary part $\partial_{6} \Omega$ of the boundary and satisfy the energy balance of the first thermodynamic principle. Therefore, one should have:

$$
\begin{array}{rll}
\underline{X}=\underline{\operatorname{grad}} \theta & \text { and } \quad \theta \in \mathcal{T} \\
R=\operatorname{div} \underline{Y} \text { in } \Omega & \text { and } & \underline{Y} \cdot \underline{n}=-g_{d} / T_{0} \text { on } \partial_{6} \Omega
\end{array}
$$

Note that the admissibility conditions (4), (5) and (6) are all decoupled for each physics, while constitutive relations (1), (2), (3) lead to an elasticity problem coupled with both the fluid and the temperature, and a thermal problem coupled with the fluid quantities. Finally, the fluid flow is a stand-alone problem. For this steady state problem, this is therefore a one-way (or weak) coupling. Note that it would become a strong coupling for the transient case [25].

\section{Scale expansion of the unknowns and homog- enization procedure}

The homogenization of the behavior model for a material that exhibits an heterogeneous microstructure usually aims to replace the micro description of a representative volume element (RVE) with heterogeneities, by an equivalent homogeneous volume filled with an homogenized material. The equivalence is often understood as an identical energy when both are subjected to uniform loadings.

Several approaches can be used, and the one that is selected herein is related to the periodic homogenization in order to avoid artificial micro edge effects when testing the response of the heterogeneous RVE, which is in this case an elementary 'cell' that can reproduce the whole microstructure by tiling.

Apart from providing the homogenized behavior, such procedures also lead to the so-called 'localization operators' that enable a post-treatment of the macroscopic solution to reach local values in the solution on each RVE independently.

Nevertheless, these relocalized solutions are known to get less accuracy in boundary areas where macroscopic edge effects occur, and as a consequence, when the scales are not well separated. In these cases, when a more accurate solution is required, specific procedures may be used, such as edge effect corrections $[28,1,17]$ or iterative procedures, some being based on domain decomposition [20, 21].

In the problem we are interested in, limitations of the periodic homogenization came from the lack of scale separation, because only one cell is located in the thickness direction (see Figure 2), and the axisymmetrical character of the problem that prevents the periodicity of the solution in the radial direction. Therefore, only 1-directional periodicity in the axial direction will be considered.

The resulting material behavior is not expected to give accurate local results, but one must recall that only the total flow rate is the quantity of interest for the coarse discretization. The classical periodic homogenization [27, 4] defines 
the scale ratio $\varepsilon$ as the ratio between the RVE size and the structure size. When it is assumed to be small, two variables describing the position in the considered domain are used: a 'slow' variable $\underline{x}$ and a fast variable $y$ which is rescaled as $\underline{y}=\underline{x} / \varepsilon$ and is intended to locate the position within the microscale description of the cell $Y$ (see Figure 3, left).
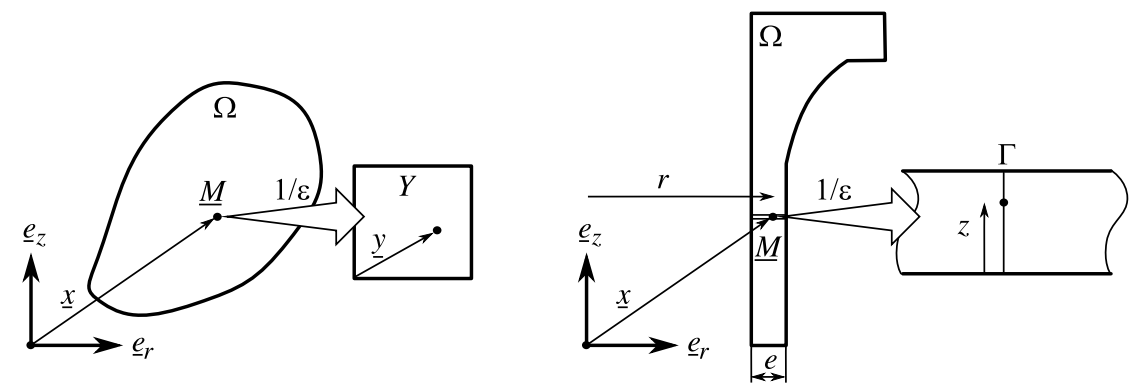

Figure 3: Two-scale description of the structure

A development with respect to $\varepsilon$ is performed for the considered unknowns. If periodicity happens only in one direction, $\underline{e}_{z}$, the fast variable should be restricted to $z=y \cdot \underline{e}_{z}$. In this case, the classical asymptotic development leads to the homogenized behavior in the axial direction only, on each vertical segment $\Gamma$ (see Figure 3, right), parametered by $r=\underline{x} \cdot \underline{e}_{r}$. This is suited to the case of a thin lamination (when the length of the segment $\Gamma$ is small with respect to the thickness $e$ ). Since this is not the case herein, we wish to maintain the homogenization on the whole cell $Y$. We therefore take some distance from the homogenization assumptions, and keep $y$ as the fast variable. As a consequence, since periodicity is not valid in the radial direction $\underline{e}_{r}$, natural boundary conditions will be prescribed directly on the cell, and edge effects will be present. In the following developments their consequences will be outlined whenever they will appear. Note that, when these boundary conditions are null fluxes, no residual appears, which is often the case for the well studied 2D periodicity conditions of composite plates, see [6] for instance, and references in [19]. This won't be the case in this article.

Moreover, with the target application of this article, we won't consider the asymptotic development of displacement field $\underline{U}$, because the solid part will be modelled directly up to the microscale, and because it does not influence the fluid and thermal part due to the loose coupling.

\subsection{Fluid problem homogenization}

The development of the pore pressure field is:

$$
p(\underline{x}, \underline{y})=\sum_{i \geqslant 0} \varepsilon^{i} p_{i}(\underline{x}, \underline{y})
$$


where all quantities are supposed to be periodic with respect to the $y$ variable. The gradient is easily obtained, once the derivation with respect to the position $\underline{M}$ is recalled as: $\frac{\partial}{\partial \underline{M}}=\frac{\partial}{\partial \underline{x}}+\frac{1}{\varepsilon} \frac{\partial}{\partial \underline{y}}$ and using subscripts $x$ and $y$ to denote derivatives with respect to the corresponding variables $\underline{x}$ and $\underline{y}$ :

$$
\underline{Z}(\underline{x}, \underline{y})=\frac{1}{\varepsilon} \underline{Z}_{y}\left(p_{0}\right)+\sum_{i \geqslant 0} \varepsilon^{i}\left(\underline{Z}_{x}\left(p_{i}\right)+\underline{Z}_{y}\left(p_{i+1}\right)\right)
$$

Using constitutive relation (2), where all material coefficients are supposed to be periodic functions of $\underline{y}$ only:

$$
\underline{W}(\underline{x}, \underline{y})=\frac{1}{\varepsilon} H \underline{Z}_{y}\left(p_{0}\right)+\sum_{i \geqslant 0} \varepsilon^{i} \underline{W}_{i}(\underline{x}, \underline{y})
$$

where $\underline{W}_{i}=H\left(\underline{Z}_{x}\left(p_{i}\right)+\underline{Z}_{y}\left(p_{i+1}\right)\right)$.

Boundary conditions are supposed to be smooth, and to depend only on the slow variable, i.e. $p_{d}(\underline{x}), w_{d}(\underline{x})$.

Fluid admissibility conditions (4) can be developed as well. The expansion of the divergence reads:

$$
\begin{aligned}
\operatorname{div} \underline{W}=\frac{1}{\varepsilon^{2}} \operatorname{div}_{y} H \underline{Z}_{y}\left(p_{0}\right)+\frac{1}{\varepsilon}\left(\operatorname{div}_{x} H \underline{Z}_{y}\left(p_{0}\right)+\operatorname{div}_{y} \underline{W}_{0}\right)+ \\
+\sum_{i \geqslant 0} \varepsilon^{i}\left(\operatorname{div}_{x} \underline{W}_{i}+\underline{\operatorname{div}}_{y} \underline{W}_{i+1}\right)
\end{aligned}
$$

The various terms in this development can be identified with respect to the order of the scale ratio $\varepsilon$. This is done in the following. At this point, one can notice that the fluid cell is not defined at the reinforcement positions since these are impervious, Figure 4. Therefore, null fluid flux is prescribed at the ceramic / steel interface $\partial_{4} Y$.

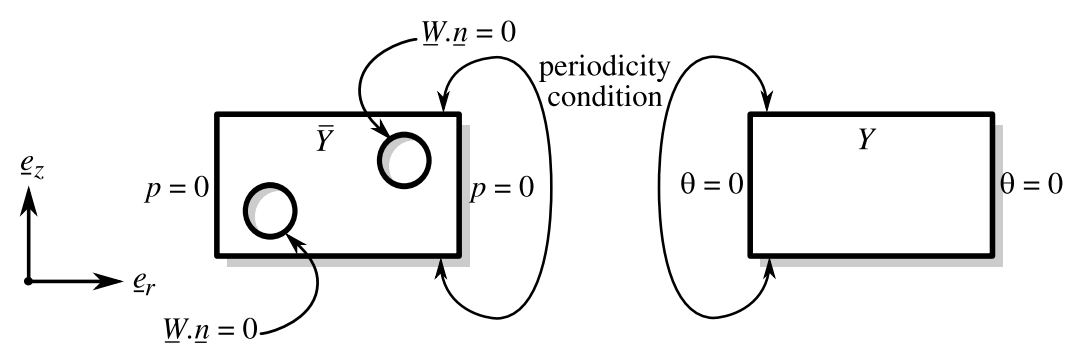

Figure 4: Fluid cell problem $(\bar{Y})$ and thermal cell problem $(Y)$

$\varepsilon^{-2}$ terms to get information on $p_{0}$. This problem reads:

$$
\operatorname{div}_{y} H \underline{Z}_{y}\left(p_{0}\right)=0,\left.\quad H \underline{Z}_{y}\left(p_{0}\right) \cdot \underline{n}\right|_{\partial_{4} Y}=0,\left.\quad p_{0}\right|_{\partial_{3} Y}=p_{d}
$$


which indicates that $p_{0}$ is a function of $\underline{x}$ only. Therefore $\underline{Z}_{y}\left(p_{0}\right)=0$ and $p_{0}$ and $\underline{Z}_{x}\left(p_{0}\right)$ will be denoted with $p_{M}$ and $\underline{Z}_{M}$, the macroscopic pore pressure and pressure gradient.

$\varepsilon^{-1}$ terms to get information on $p_{1}$. The local pore pressure $p_{1}(\underline{x}, y)$ possesses periodicity property on $\partial_{\text {per }} Y$. In our case, $\partial_{\text {per }} Y$ is the upper and lower part of the boundary $\partial Y$, see Figure 4. On the remaining part of the cell boundary, direct boundary conditions have to be prescribed.

The space of fields $p_{1}$ that verify periodicity conditions on $\partial_{\text {per }} Y$ and $p_{1}=0$ on $\partial_{3} Y$ is denoted with $\mathcal{P}_{0}^{\#}$. The problem is therefore:

$$
\begin{array}{r}
p_{1} \in \mathcal{P}_{0}^{\#} \\
\operatorname{div}_{y} \underline{W}_{0}=0 \text { in } Y \text { and } \quad \underline{W}_{0} \cdot \underline{n}=w_{d} \text { on } \partial_{4} Y
\end{array}
$$

The variational formulation corresponding to $(12 \mathrm{~b})$ is:

$$
\forall p_{1}^{\star} \in \mathcal{P}_{0}^{\#}, \int_{Y} p_{1}^{\star} \operatorname{div}_{y} \underline{W}_{0} d Y=0=-\int_{Y} \underline{\operatorname{grad}}_{y} p_{1}^{\star} \cdot \underline{W}_{0} d Y+\int_{\partial Y} p_{1}^{\star} \underline{W}_{0} \cdot \underline{n} d S
$$

The integral on the boundary is null on $\partial_{3} Y$. With $\underline{W}_{0}=H\left(\underline{Z}_{M}+\underline{Z}_{y}\left(p_{1}\right)\right)$, which is periodic in axial direction, the integral on $\partial_{\text {per }} Y$ is null as well. Therefore, there only remains the residual term:

$$
\int_{\partial_{4} Y} p_{1}^{\star} w_{d} d S
$$

This term arises from the direct imposition of boundary conditions on the cell boundary. It traduces an edge effect in the cell if $w_{d} \neq 0$. The modelling choice we select here is to discard this term; therefore, prescribed fluxes are taken into account only at the macroscopic scale.

Therefore, one has $p_{1} \in \mathcal{P}_{0}^{\#}$ such that:

$$
\forall p_{1}^{\star} \in \mathcal{P}_{0}^{\#}, \int_{Y} \underline{\operatorname{grad}}_{y} p_{1}^{\star} \cdot H \underline{\operatorname{grad}}_{y} p_{1} d Y=-\int_{Y} \underline{\operatorname{grad}}_{y} p_{1}^{\star} \cdot H \underline{Z}_{M} d Y
$$

This problem leads to a unique solution in pore pressure gradient which depends linearly on the macroscopic gradient:

$$
\underline{Z}_{y}\left(p_{1}\right)=\mathbf{L}_{F}(\underline{y}) \underline{Z}_{M}
$$

where $\mathbf{L}_{F}$ is the localization operator for the fluid problem.

$\varepsilon^{0}$ terms to get information on the homogenized behavior. This problem reads:

$$
\operatorname{div}_{x} \underline{W}_{0}+\operatorname{div}_{y} \underline{W}_{1}=0
$$

with $\underline{W}_{1}$ periodic and verifying $\underline{W}_{1} \cdot \underline{n}=0$ on $\partial_{4} Y$. Here, we choose to express the average of (17) on the elementary cell. We define the average on the 3D 
axisymmetric cell $Y$ (whose radial section is $\mathcal{Y}$, see Figure 5) as the componentper-component average in the local basis $\left(\underline{e}_{r}, \underline{e}_{\theta}, \underline{e}_{z}\right)$, which is equivalent to the average on section $\mathcal{Y}$ weighted by the current radius $r$ :

$$
\langle\bullet\rangle=\frac{1}{\operatorname{vol}(Y)} \int_{Y} \bullet d Y=\frac{1}{\int_{\mathcal{Y}} r d \mathcal{Y}} \int_{\mathcal{Y}} \bullet r d \mathcal{Y}
$$

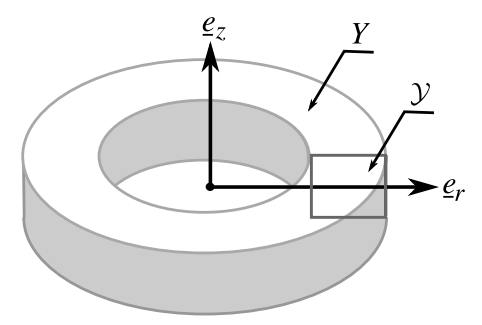

Figure 5: 3D cell $Y$ and its radial section $\mathcal{Y}$

Therefore, with:

$$
\int_{Y} \operatorname{div}_{y} \underline{W}_{1} d Y=\int_{\partial Y} \underline{W}_{1} \cdot \underline{n} d S=\int_{\partial_{3} Y} \underline{W_{1}} \cdot \underline{n} d S
$$

(17) leads to:

$$
\operatorname{div}_{x}\left\langle\underline{W}_{0}\right\rangle=-\frac{1}{\operatorname{vol}(Y)} \int_{\partial_{3} Y} \underline{W_{1}} \cdot \underline{n} d S
$$

This term is also a residual term due to edge effect. We choose again to discard it. Therefore, the macroscopic Darcy velocity is $\underline{W}_{M}=\left\langle\underline{W}_{0}\right\rangle$ and its expression is:

$$
\underline{W}_{M}=\left\langle\underline{W}_{0}\right\rangle=\left\langle H\left(\underline{Z}_{M}+\underline{Z}_{y}\left(p_{1}\right)\right)\right\rangle=\left\langle H\left(\mathbf{I}+\mathbf{L}_{F}\right)\right\rangle \underline{Z}_{M}
$$

The corresponding homogenized permeability tensor is:

$$
\mathbf{H}_{M}=\left\langle H\left(\mathbf{I}+\mathbf{L}_{F}\right)\right\rangle
$$

\subsection{Thermal problem homogenization}

The development of the temperature field and its gradient are:

$$
\begin{aligned}
\theta(\underline{x}, \underline{y}) & =\sum_{i \geqslant 0} \varepsilon^{i} \theta_{i}(\underline{x}, \underline{y}) \\
\underline{X}(\underline{x}, \underline{y}) & =\frac{1}{\varepsilon} \underline{X}_{y}\left(\theta_{0}\right)+\sum_{i \geqslant 0} \varepsilon^{i}\left(\underline{X}_{x}\left(\theta_{i}\right)+\underline{X}_{y}\left(\theta_{i+1}\right)\right)
\end{aligned}
$$

Constitutive relations (2) and (3) read:

$$
\begin{aligned}
& \underline{Y}(\underline{x}, \underline{y})=\frac{1}{\varepsilon} \frac{k}{T_{0}} \underline{X}_{y}\left(\theta_{0}\right)+\sum_{i \geqslant 0} \varepsilon^{i} \underline{Y}_{i}(\underline{x}, \underline{y}) \\
& R(\underline{x}, \underline{y})=-\frac{1}{\varepsilon} H \frac{\rho_{F} c_{F}}{T_{0}} \underline{X}_{y}\left(\theta_{0}\right) \cdot\left(\underline{Z}_{M}+\underline{Z}_{y}\left(p_{1}\right)\right)+\sum_{i \geqslant 0} \varepsilon^{i} R_{i}(\underline{x}, \underline{y})
\end{aligned}
$$


where, in particular:

$$
\begin{aligned}
\underline{Y}_{i}= & \frac{k}{T_{0}}\left(\underline{X}_{x}\left(\theta_{i}\right)+\underline{X}_{y}\left(\theta_{i+1}\right)\right) \\
R_{0}= & -\frac{\rho_{F} c_{F}}{T_{0}}\left(\underline{Z}_{M}+\underline{Z}_{y}\left(p_{1}\right)\right) \cdot\left(\underline{X}_{x}\left(\theta_{0}\right)+\underline{X}_{y}\left(\theta_{1}\right)+\underline{X}_{y}\left(\theta_{0}\right)\right)+ \\
& -\left(\frac{1}{T_{0}}-3 \alpha_{F}\right) H\left(\underline{Z}_{M}+\underline{Z}_{y}\left(p_{1}\right)\right)^{2}
\end{aligned}
$$

With a similar expression as previously for the fluid, the divergence can be developed for the heat equation, and the various terms in the development are identified as in the following.

$\varepsilon^{-2}$ terms to get information on $\theta_{0}$. This problem reads:

$$
\operatorname{div}_{y} \frac{k}{T_{0}} \underline{X}_{y}\left(\theta_{0}\right)=0,\left.\quad \frac{k}{T_{0}} \underline{X}_{y}\left(\theta_{0}\right) \cdot \underline{n}\right|_{\partial_{6} Y}=0,\left.\quad \theta_{0}\right|_{\partial_{5} Y}=\theta_{d}
$$

which indicates that $\theta_{0}$ is a function of $\underline{x}$ only. Therefore $\underline{X}_{y}\left(\theta_{0}\right)=0$ and $\theta_{0}$ and $\underline{X}_{x}\left(\theta_{0}\right)$ will be denoted with $\theta_{M}$ and $\underline{X}_{M}$, the macroscopic temperature and temperature gradient.

$\varepsilon^{-1}$ terms to get information on $\theta_{1}$. As for the fluid, the space of regular fields $\theta_{1}$ that verify periodicity conditions on $\partial_{\text {per }} Y$ and $\theta_{1}=0$ on $\partial_{5} Y$ is denoted with $\mathcal{T}_{0}^{\#}$. The problem is therefore:

$$
\begin{array}{r}
\theta_{1} \in \mathcal{T}_{0}^{\#} \\
\operatorname{div}_{y} \underline{Y}_{0}=0 \text { in } Y \quad \text { and } \quad \underline{Y}_{0} \cdot \underline{n}=g_{d} \text { on } \partial_{6} Y
\end{array}
$$

Then, the variational formulation corresponding to (27b) is:

$$
\forall \theta_{1}^{\star} \in \mathcal{T}_{0}^{\#}, \int_{Y} \theta_{1}^{\star} \operatorname{div}_{y} \underline{Y}_{0} d Y=0=-\int_{Y} \underline{\operatorname{grad}}_{y} \theta_{1}^{\star} \cdot \underline{Y}_{0} d Y+\int_{\partial Y} \theta_{1}^{\star} \underline{Y}_{0} \cdot \underline{n} d S
$$

As previously, the integral on the boundary is null on $\partial_{5} Y$ and $\partial_{\text {per }} Y$. The residual term which will be neglected here is:

$$
\int_{\partial_{6} Y} \theta_{1}^{\star} g_{d} d S
$$

Therefore, one has $\theta_{1} \in \mathcal{T}_{0}^{\#}$ such that:

$$
\forall \theta_{1}^{\star} \in \mathcal{T}_{0}^{\#}, \int_{Y} \underline{\operatorname{grad}}_{y} \theta_{1}^{\star} \cdot \frac{k}{T_{0}} \underline{\operatorname{grad}}_{y} \theta_{1} d Y=-\int_{Y} \underline{\operatorname{grad}}_{y} \theta_{1}^{\star} \cdot \frac{k}{T_{0}} \underline{Z}_{M} d Y
$$

As previously, the unique solution in temperature gradient depends linearly on its macroscopic gradient:

$$
\underline{X}_{y}\left(\theta_{1}\right)=\mathbf{L}_{T}\left(\underline{y}_{)} \underline{X}_{M}\right.
$$

where $\mathbf{L}_{T}$ is the localization operator for the thermal problem. 
$\varepsilon^{0}$ terms to get information on the homogenized behavior. This problem reads:

$$
\operatorname{div}_{x} \underline{Y}_{0}+\operatorname{div}_{y} \underline{Y}_{1}=R_{0}
$$

with $\underline{Y}_{1}$ periodic and verifying $\underline{Y}_{1} \cdot \underline{n}=0$ on $\partial_{6} Y$. Here, we choose again to express the average of (32) on the elementary cell. Therefore, with:

$$
\int_{Y} \operatorname{div}_{y} \underline{Y}_{1} d Y=\int_{\partial Y} \underline{Y}_{1} \cdot \underline{n} d S=\int_{\partial_{5} Y} \underline{Y}_{1} \cdot \underline{n} d S
$$

(32) leads to:

$$
\operatorname{div}_{x}\left\langle\underline{Y}_{0}\right\rangle=\left\langle R_{0}\right\rangle-\frac{1}{\operatorname{vol}(Y)} \int_{\partial_{3} Y} \underline{Y}_{1} \cdot \underline{n} d S
$$

The last term is the second residual due to edge effects. It will be neglected in the following.

Therefore, the macroscopic heat flux is $\underline{Y}_{M}=\left\langle\underline{Y}_{0}\right\rangle$. Its expression is:

$$
\underline{Y}_{M}=\left\langle\underline{Y}_{0}\right\rangle=\left\langle\frac{k}{T_{0}}\left(\underline{X}_{M}+\underline{X}_{y}\left(\theta_{1}\right)\right\rangle=\left\langle\frac{k}{T_{0}}\left(\mathbf{I}+\mathbf{L}_{T}\right)\right\rangle \underline{X}_{M}\right.
$$

and the homogenized conductivity tensor is:

$$
\mathbf{k}_{M}=\left\langle k\left(\mathbf{I}+\mathbf{L}_{T}\right)\right\rangle
$$

Moreover, the macroscopic dissipation is $R_{M}=\left\langle R_{0}\right\rangle$. Its expression is:

$$
\begin{aligned}
\left\langle R_{0}\right\rangle= & -\underline{Z}_{M} \cdot \underbrace{\left\langle\left[H\left(\mathbf{I}+\mathbf{L}_{F}\right)\right]^{T} \frac{\rho_{F} c_{F}}{k}\left[\frac{k}{T_{0}}\left(\mathbf{I}+\mathbf{L}_{T}\right)\right]^{T}\right\rangle}_{\mathbf{c}_{M}} \underline{X}_{M}+ \\
& -\underline{Z}_{M} \cdot \underbrace{\left\langle\left[H\left(\mathbf{I}+\mathbf{L}_{F}\right)\right]^{T} \frac{1}{H}\left(\frac{1}{T_{0}}-3 \alpha_{F}\right)\left[H\left(\mathbf{I}+\mathbf{L}_{F}\right)\right]^{T}\right\rangle}_{\mathbf{d}_{M}} \underline{Z}_{M} \\
= & -\underline{Z}_{M} \cdot \underline{\mathbf{c}}_{M} \underline{X}_{M}-\underline{Z}_{M} \cdot \mathbf{d}_{M} \underline{Z}_{M}
\end{aligned}
$$

The macroscopic effect of the convection is stored in tensor $\mathbf{c}_{M}$ while the macroscopic dissipation lies in tensor $\mathbf{d}_{M}$.

\section{Field and scale transfer for the coupled prob- lem solution}

Due to the choice of modeling for the aforementioned filtration problem, the coarse mesh for fluid and thermal problems is assumed to capture the macroscopic quantities only. Therefore homogenized behavior are used for each of these physics.

On the other hand, the fine mesh for the elastic problem may capture the local micro stresses and the micro heterogeneous behavior is used for it. 


\subsection{Fluid problem}

The homogenized fluid problem consists in searching for fluid unknowns satisfying to:

- the constitutive relation:

$$
\underline{W}_{M}=\mathbf{H}_{M} \underline{Z}_{M}
$$

- the admissibility conditions:

$$
\begin{array}{rll}
\underline{Z}_{M}=\underline{\operatorname{grad}} p_{M} & \text { and } & p_{M} \in \mathcal{P} \\
\operatorname{div} \underline{W}_{M}=0 \text { in } \Omega & \text { and } & \underline{W_{M}} \cdot \underline{n}=w_{d} \text { on } \partial_{4} \Omega
\end{array}
$$

Introducing $\mathcal{P}_{0}$ the vector space corresponding to $\mathcal{P}$, the variational formulation of the second condition reads:

$$
\forall p^{\star} \in \mathcal{P}_{0}, \int_{\Omega} \underline{\operatorname{grad}} p^{\star} \cdot \underline{W}_{M} d \Omega=\int_{\partial_{4} \Omega} p^{\star} w_{d} d S
$$

Together with the constitutive relation, the problem is to find $p_{M} \in \mathcal{P}$ such that:

$$
\forall p^{\star} \in \mathcal{P}_{0}, \int_{\Omega} \underline{\operatorname{grad}} p^{\star} \cdot \mathbf{H}_{M} \underline{\operatorname{grad}} p_{M} d \Omega=\int_{\partial_{4} \Omega} p^{\star} w_{d} d S
$$

Once discretized with finite elements, this leads to a linear system of the form:

$$
\boldsymbol{H} \boldsymbol{p}_{M}=\boldsymbol{w}
$$

where $\boldsymbol{H}$ is the (macroscopic) permeability matrix and $\boldsymbol{w}$ the generalized fluid flux.

For this macroscopic problem, the boundary conditions are prescribed classically. No corresponding term emerges from the underlying microscale because residual terms in (14) and (20) have been neglected.

With a known pore pressure, the thermal problem can now be solved.

\subsection{Thermal problem}

The homogenized thermal problem consists in searching for thermic unknowns satisfying to:

- the constitutive relations:

$$
\begin{aligned}
& \underline{Y}_{M}=\frac{\mathbf{k}_{M}}{T_{0}} \underline{X}_{M} \\
& R_{M}=-\underline{Z}_{M} \cdot \mathbf{c}_{M} \underline{X}_{M}-\underline{Z}_{M} \cdot \mathbf{d}_{M} \underline{Z}_{M}
\end{aligned}
$$

- the admissibility conditions:

$$
\begin{array}{rll}
\underline{X}_{M}=\underline{\operatorname{grad}} \theta_{M} & \text { and } \quad \theta_{M} \in \mathcal{T} \\
\operatorname{div} \underline{Y}_{M}=R_{M} \text { in } \Omega & \text { and } & \underline{Y}_{M} \cdot \underline{n}=g_{d} \text { on } \partial_{6} \Omega
\end{array}
$$


Introducing $\mathcal{T}_{0}$ the vector space corresponding to $\mathcal{T}$, the variational formulation of the second previous condition reads:

$$
\forall \theta^{\star} \in \mathcal{T}_{0}, \int_{\Omega}\left(\theta^{\star} R_{M}+\underline{\operatorname{grad}} \theta^{\star} \cdot \underline{Y}_{M}\right) d \Omega=\int_{\partial_{6} \Omega} \theta^{\star} g_{d} d S
$$

Together with the constitutive relations, the problem is to find $\theta_{M} \in \mathcal{T}$ such that:

$$
\begin{aligned}
\forall \theta^{\star} \in \mathcal{T}_{0}, \int_{\Omega}\left(-\theta^{\star} \underline{Z}_{M} \cdot \mathbf{c}_{M} \underline{\operatorname{grad}} \theta_{M}+\underline{\operatorname{grad}} \theta^{\star} \cdot \frac{\mathbf{k}_{M}}{T_{0}} \underline{\operatorname{grad}} \theta_{M}\right) d \Omega \\
=\int_{\Omega} \theta^{\star} \underline{Z}_{M} \cdot \mathbf{d}_{M} \underline{Z}_{M} d \Omega+\int_{\partial_{6} \Omega} \theta^{\star} g_{d} d S
\end{aligned}
$$

Once discretized with finite elements, this leads to a linear system of the form:

$$
\left(-\boldsymbol{c}_{F}+\boldsymbol{c}\right) \boldsymbol{\theta}_{M}=\boldsymbol{B}_{R}^{T} \bar{r}+\boldsymbol{g}
$$

where $\boldsymbol{c}_{F}$ is a non-symmetric matrix depending on the fluid solution $\underline{Z}_{M}$ and representing the advection of the heat due to the flow, $\boldsymbol{c}$ is the conductivity matrix, $g$ is the generalized thermal flux, $\boldsymbol{B}_{R}$ is a classical matrix used to compute the generalized flux that equilibrates a given source, here the viscosity dissipation $\bar{r}=\underline{Z}_{M}^{T} \boldsymbol{c}_{M} \underline{Z}_{M}$.

As previously, for this macroscopic problem, no term due to the boundary conditions emerges from the underlying microscale because residual terms in (29) and (34) have been neglected.

Provided that both the fluid an thermal macroscopic problems are solved, the microscopic elastic problem can be stated.

\subsection{Solid problem}

With given micro fluid and thermal known quantities, $p$ and $\theta$, the micro elastic problem consists in:

- the constitutive relation:

$$
\boldsymbol{\sigma}=\mathbf{D} \boldsymbol{\varepsilon}-b p \mathbf{I}-3 \alpha K_{b} \theta \mathbf{I}
$$

- the admissibility conditions:

$$
\begin{array}{lll}
\varepsilon=\left(\frac{\partial \underline{U}}{\partial \underline{M}}\right)_{\text {sym }} & \text { and } \quad \underline{U} \in \mathcal{U} \\
\underline{\operatorname{div}} \boldsymbol{\sigma}=\underline{0} \text { in } \Omega & \text { and } & \boldsymbol{\sigma} \underline{n}=\underline{F}_{d} \text { on } \partial_{2} \Omega
\end{array}
$$

Introducing $\mathcal{U}_{0}$ the vector space corresponding to $\mathcal{U}$, the variational formulation of the second previous condition reads:

$$
\forall \underline{U}^{\star} \in \mathcal{U}_{0}, \int_{\Omega} \operatorname{Tr}\left[\varepsilon\left(\underline{U}^{\star}\right) \boldsymbol{\sigma}\right] d \Omega=\int_{\partial_{2} \Omega} \underline{U}^{\star} \cdot \underline{F}_{d} d S
$$


Together with the constitutive relation, the problem is to find $\underline{U} \in \mathcal{U}$ such that:

$$
\begin{aligned}
\forall \underline{U}^{\star} \in \mathcal{U}_{0}, \int_{\Omega} \operatorname{Tr}\left[\varepsilon\left(\underline{U}^{\star}\right) \mathbf{D} \varepsilon(\underline{U})\right] d \Omega \\
\quad=\int_{\Omega} \operatorname{Tr}\left[\varepsilon\left(\underline{U}^{\star}\right)\left(b p+3 \alpha K_{b} \theta\right) \mathbf{I}\right] d \Omega+\int_{\partial_{2} \Omega} \underline{U}^{\star} \cdot \underline{F}_{d} d S
\end{aligned}
$$

Once discretized with finite elements, this leads to a linear system of the form:

$$
\boldsymbol{K} \boldsymbol{u}=\boldsymbol{B}_{\sigma}^{T} \overline{\boldsymbol{\sigma}}+\boldsymbol{f}
$$

where $\boldsymbol{K}$ is the stiffness matrix, $\boldsymbol{f}$ the generalized forces and $\boldsymbol{B}_{\sigma}$ the classical operator that computes the generalized forces that balance an internal stress, here $\overline{\boldsymbol{\sigma}}=\left(b p+3 \alpha K_{b} \theta\right) \mathbf{I}$.

The last difficulty is to get $\overline{\boldsymbol{\sigma}}$ from macro quantities $p_{M}$ and $\theta_{M}$ defined on a different mesh. This is the topic of the next Section.

\subsection{Field transfer}

With a given pore pressure $p$ and temperature $\theta$ defined on the fluid and thermic mesh, interpreted as macroscopic fields, the objective is to get the microscopic stress $\overline{\boldsymbol{\sigma}}$. This last quantity is defined at the integration points of the solid mesh. The transfer operator designed in [12] is based on a variational formulation that embeds an energy equivalence. Its feature is to deal with fields defined at integration points of non matching meshes without any master / slave approach, i.e. with a symmetric treatment of field transfer in each direction. Its ability to deal with strongly and fully coupled problems such as thermo-viscoelasticity or thermo-poroelasticity has been exemplified in [12, 25], as well as its adaptation to transfer between different time discretizations in [24].

The pore pressure and temperature to be transferred can easily be expressed at the integration points of the fluid and thermic mesh. Once they have been projected, the resulting fields are still macroscopic ones.

The first approach is to use an identity localization operator, i.e. to consider that the projected fields are also the microscopic fields. If $\mathcal{P}$ is the projector, the internal stress is therefore:

$$
\overline{\boldsymbol{\sigma}}=\left(b \mathcal{P} p+3 \alpha K_{b} \mathcal{P} \theta\right) \mathbf{I}
$$

Other strategies are possible. For instance, if one gets the averaged gradients $\underline{Z}_{M}$ and $\underline{X}_{M}$ on each cell (they can be extracted from fields $\mathcal{P} p$ and $\mathcal{P} \theta$ ) the relocalization operators can be used to get:

$$
\overline{\boldsymbol{\sigma}}=\left[b\left(\mathcal{P} p+\mathbf{L}_{F} \underline{Z}_{M}\right)+3 \alpha K_{b}\left(\mathcal{P} \theta+\mathbf{L}_{T} \underline{X}_{M}\right)\right] \mathbf{I}
$$

on each cell. Note that with this approach, macro fields $\mathcal{P} p$ and $\mathcal{P} \theta$ are not uniform on each cell. Indeed, due to the lack of scale separation, the edge effects 
are hopefully recovered (at least partly since in any case, the approximation considering the residual terms (14), (20), (29) and (34) as null has been used) by the evolution of these fields at the cell level. This approach is currently under testing, and only the first basic approach will be used in the following.

A simple projection from fluid and thermic mesh onto solid mesh is therefore required. The extension of the previously mentioned projector to the axisymmetric case is easy to perform:

- If the field to project is defined at integration points, its values are extrapolated to the nodes of each element independently (with a least square approach) to allow its interpolation on each element;

- The resulting field is projected to the elements of the target mesh using an average extraction procedure (similar to a weighted residual). For instance if $p_{F}$ is defined on the elements of the mesh $\Omega_{F}$, its projection $p_{S}$ onto the mesh $\Omega_{S}$ is such that:

$$
\forall p_{S}^{\star} \text { defined on } \Omega_{S}, \int_{\Omega_{S}} p_{S} p_{S}^{\star} d \Omega=\int_{\Omega_{F}} p_{F} p_{S}^{\star} d \Omega
$$

Unlike classical mortar approaches [5, 3, 7], dealing with element per element interpolations for $p_{S}^{\star}$ also avoids the globality of the projection (55). It can be performed on a 1-layer element neighborhood basis, due to the cross product on the right hand side;

- Finally, the resulting field $p_{S}$ can be interpolated to the integration points of the target mesh, for each element independently.

The overall procedure is denoted with the application of the projector $\mathcal{P}$. Concerning axisymmetric problems, and for scalar fields, the integral in (55) should be interpreted as the integral on the $3 \mathrm{D}$ volume. On the radial section, this is transformed into:

$$
\forall p_{S}^{\star} \text { defined on } \Omega_{S}, \int_{\Omega_{S}} p_{S} p_{S}^{\star} r d S=\int_{\Omega_{F}} p_{F} p_{S}^{\star} r d S
$$

Therefore, the axisymmetric version can be interpreted as a weighted generalization of the previously used transfer, using the current radius $r$ as weight.

\section{$5 \quad$ Numerical results}

The goal of this Section is to assess the feasibility of the proposed strategy. To quantify the results obtained in each case, a 'reference' solution is also computed, on the single mesh for all the involved physics. The convergence when refining the solid mesh is also tested, for which a fixed reference solution is obtained with a direct coupled approach on a single refined mesh. 


\subsection{Test case description}

The considered problem is the previously mentioned reinforced filtration device. The inflow has the pressure $p_{d}$ and the relative temperature $\theta_{d}$, while the outflow has null pressure and temperature. Apart from the symmetry condition on half of the radial section, the upper part of the boundary is assembled with an external metallic part, supposed to be impervious and for which the temperature decreases radially with the following model:

$$
\theta(r)=\theta_{d}(1-\zeta) e^{-6 \zeta} \quad \text { with } \quad \zeta=\left(r-r_{i}\right) /\left(r_{e}-r_{i}\right)
$$

Concerning the solid part, the external pressures lead to mechanical forces, and the upper part of the boundary is clamped. These boundary conditions are depicted in Figure 6.

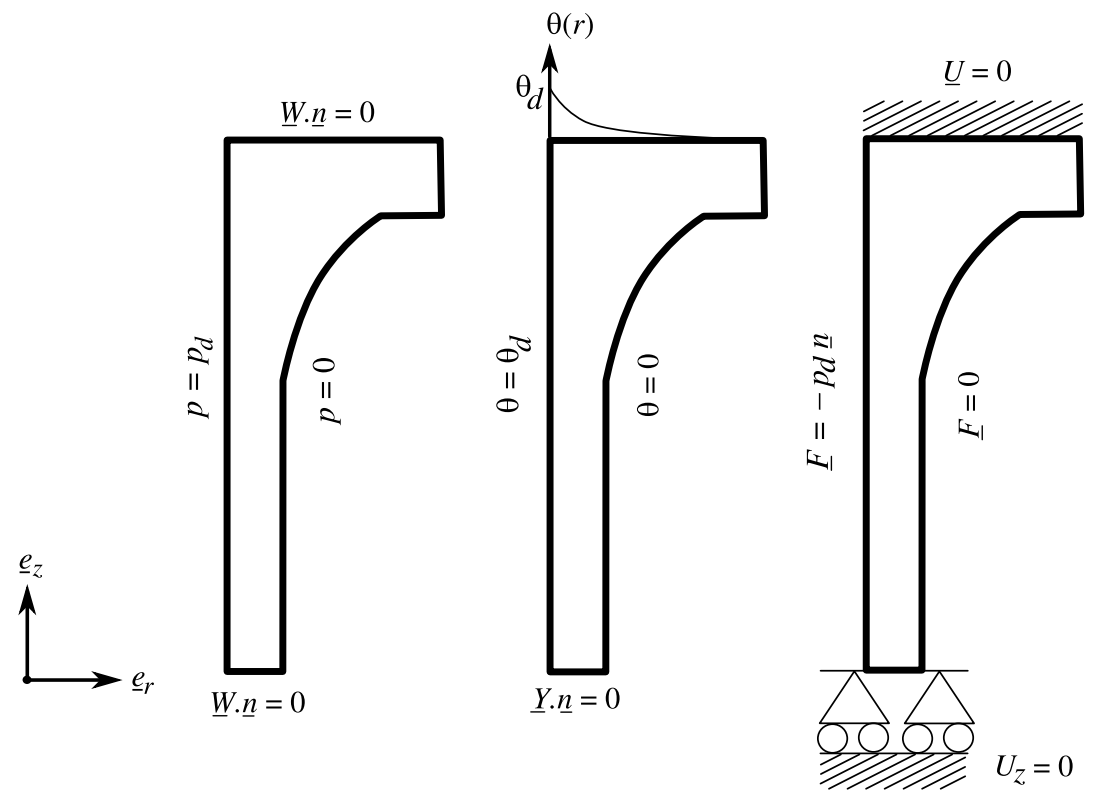

Figure 6: Fluid, thermal and structural problems

The characteristic values for the considered problem are reported in Table 3. The Nusselt number $\mathcal{N}_{u}$ is defined as the ratio of the heat convectively transported by the fluid and the heat supplied by conduction. The Brinkman number $\mathcal{B}_{r}$ is the ratio of the heat source due to the viscous dissipation and the heat supplied by conduction. Depending on their relative value with respect to 1 , for a particular application, the last two nonlinear terms in $R$ (3) can sometimes be neglected. Their order of magnitude can be estimated as:

$$
\mathcal{N}_{u}=\frac{\rho_{F} c_{F}}{k} H p_{d} \quad \text { and } \quad \mathcal{B}_{r}=\frac{H\left(\frac{1}{T_{0}}-3 \alpha_{F}\right)}{\frac{k}{T_{0}} \theta_{d}} p_{d}^{2}
$$




\begin{tabular}{ll}
\hline Length & $L=2.5 \mathrm{~m}$ \\
Inner diameter & $D=1 \mathrm{~m}$ \\
Thickness & $e=0.25 \mathrm{~m}$ \\
Wire diameter & $d=5.6 \mathrm{~cm}$ \\
Implantation diameters & $D_{1}=1.11 \mathrm{~m}$ and $D_{2}=1.39 \mathrm{~m}$ \\
Pitch & $l=13.9 \mathrm{~cm}$ \\
\hline Inflow pressure & $p_{d}=1.5 \mathrm{MPa}$ \\
Inflow relative temperature & $\theta_{d}=80 \mathrm{~K}$ \\
\hline Nusselt number (radial direction) & $\mathcal{N}_{u}=18.5$ \\
Brinkman number (radial direction) & $\mathcal{B}_{r}=0.064$ \\
\hline
\end{tabular}

Table 3: Geometrical characteristics and boundary conditions

The small value of Brinkman number indicates that the dissipation term in (3) could have been neglected, but the value of Nusselt number confirms that the convective term should be taken into account.

Several simulations are performed: the fluid and thermic mesh is kept constant, see Figure 2, with $n=189$ 3-node triangles, and several solid meshes are tested: $n_{S}=2084,4810,7682,10842,15358$ 6-node straight-edge elements. The solutions will be referred to as cases 1 to 5 , respectively.

\subsection{Localization operators and homogenized material co- efficients}

The relocalization operators are computed numerically on a generic cell, meshed at the microscale. They are illustrated in Figures 7 and 8 (the post-treatment has been performed with the plotting facilities of gmsh ${ }^{\circledR}$ software [26]). The first Figure (lower part) gives the localized gradients $\underline{Z}_{y}\left(p_{1}\right)$ and $\underline{X}_{y}\left(\theta_{1}\right)$ for a unitary macro gradient in the radial direction $\underline{e}_{r}$, while the second Figure corresponds to a unitary macro gradient in the axial direction $\underline{e}_{z}$. In the present case, direct imposition of boundary conditions on the cell leads to a unique localized pore pressure $p_{1}$ and temperature $\theta_{1}$. Therefore, two additional relocalization operators $\tilde{\mathbf{L}}_{F}$ and $\tilde{\mathbf{L}}_{T}$ can be defined with $p_{1}=\tilde{\mathbf{L}}_{F} \underline{Z}_{M}$ and $\theta_{1}=\tilde{\mathbf{L}}_{T} \underline{X}_{M}$. These last two operators are also illustrated on the upper part of Figures 7 and 8. Each of these Figures correspond to the micro mesh of case 1.

The homogenized material characteristics given by the finest cell mesh (case 5) are recalled in Table 4.

\subsection{Coupled problems results}

Concerning numerical integration, with these elements and an axisymmetric formulation, polynomials of order 3 have to be integrated for the stiffness. A cubature scheme with $n_{G}=4$ integration points is therefore a good candidate. Such a scheme that does not involve negative weights has been selected for all the elements $[18,9]$ to get identical integration point locations for the reference 

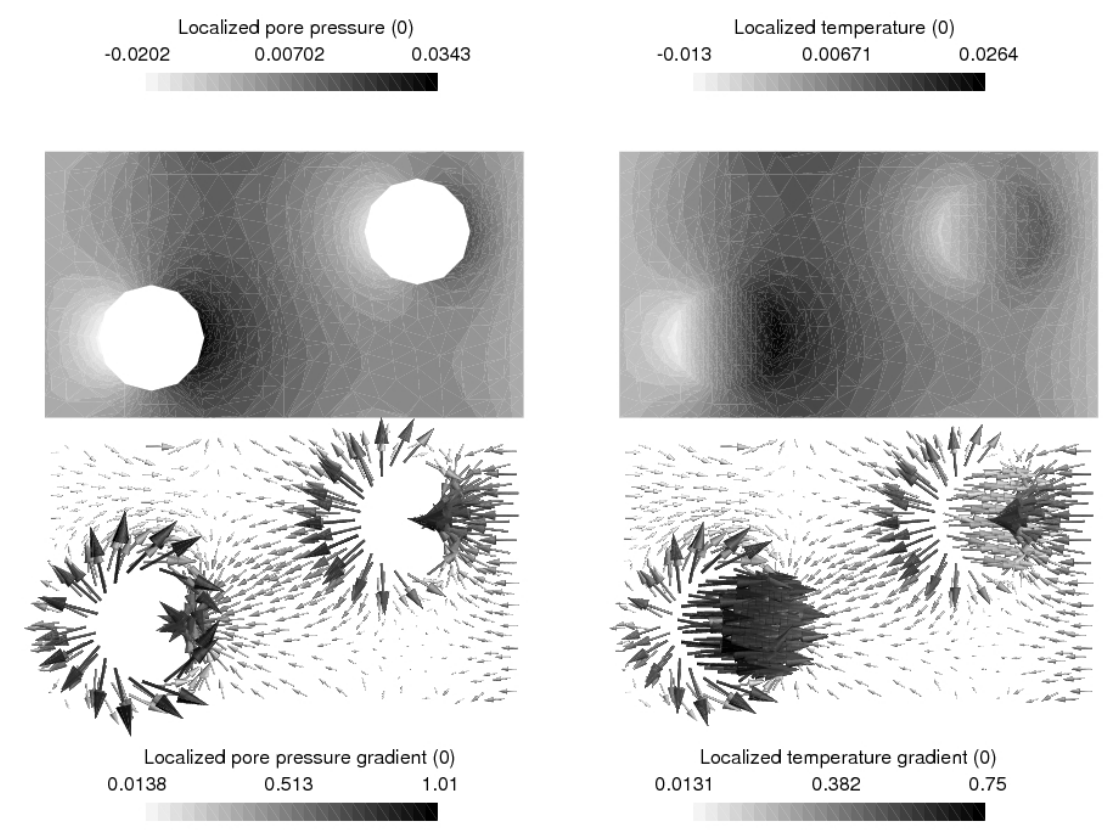

Figure 7: Localization operator for pore pressure (left) and temperature (right), corresponding to a macroscopic gradient along radial direction

solution (with conforming meshes).

When refining the solid mesh, the projected fields $p_{S}=\mathcal{P} p_{M}$ and $\theta_{S}=$ $\mathcal{P} \theta_{M}$ may vary slightly, but since the solid mesh is always finer than the other ones, this variation can be considered as negligible. The previously mentioned procedure is used to compute the loading $\bar{\sigma}$ and to solve the solid problem. Its post-treatment leads to the maximal principal stress $\sigma$.

A reference computation is performed by selecting the solid mesh as a unique mesh for all the physics (in this case, projector $\mathcal{P}$ is the identity), and using the microscopic heterogeneous material coefficient fields. This produces 'reference' pore pressure $p_{\text {ref }}$, temperature $\theta_{\text {ref }}$ and maximum principal stress $\sigma_{\text {ref }}$ fields. Note that they still depend on the refinement selected.

Several indicators can be build: for the fluid and thermic problems,

$$
\eta_{F}=\frac{\max _{\Omega}\left|p_{S}-p_{\text {ref }}\right|}{\max _{\Omega}\left|p_{S}\right|} \quad \text { and } \quad \eta_{T}=\frac{\max _{\Omega}\left|\theta_{S}-\theta_{\text {ref }}\right|}{\max _{\Omega}\left|\theta_{S}\right|}
$$

Note that the denominator is quasi-constant when refining the solid mesh. Since $p_{S}$ and $\theta_{S}$ are linked to the macroscopic fields, the reference fields may exhibit larger and larger variations and therefore will diverge from the macroscopic fields when refining, see Table 5 .

For the solid quantities, in order to check the convergence with respect to a fixed reference, a direct computation has been performed on a finer mesh 

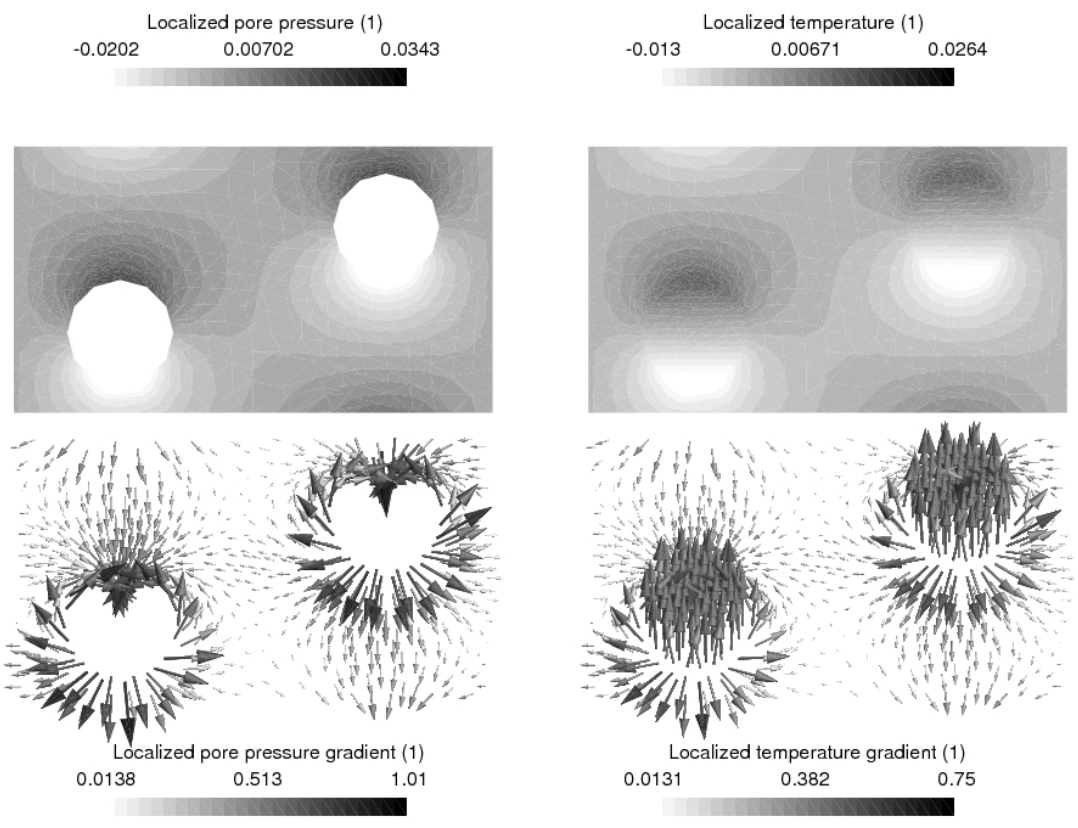

Figure 8: Localization operator for pore pressure (left) and temperature (right), corresponding to a macroscopic gradient along axial direction

(here, $n_{S}=26602$ ). This produces the 'target' maximal principal stress field $\sigma^{r}$ that can be used as a fixed reference to assess the convergence; $\max _{\Omega} \sigma^{r}=$ $0.033 \times 0.7 \mathrm{GPa}$.

The following errors are used to compare the various solutions:

$$
\eta=\frac{\left|\max _{\Omega} \sigma-\max _{\Omega} \sigma^{r}\right|}{\left|\max _{\Omega} \sigma^{r}\right|} \quad \text { and } \quad \eta_{\mathrm{ref}}=\frac{\left|\max _{\Omega} \sigma_{\mathrm{ref}}-\max _{\Omega} \sigma^{r}\right|}{\left|\max _{\Omega} \sigma^{r}\right|}
$$

Their values are reported in Table 6 .

Though convergence is not uniform, the error with the proposed strategy exhibits the same convergence order as the error of the computation with identical meshes.

Finally, Figures 9, 10 and 11 reports the temperature, pore pressure and maximal principal stress for the proposed strategy and reference of case 1 , as well as the target solution of case 6 .

\section{Conclusion}

A computational strategy has been proposed and tested for a weakly coupled problem, a steady state thermo-poroelastic problem, when different meshes and different scale descriptions are used for the different physics. 


\begin{tabular}{ll}
\hline Conductivity tensor & $\mathbf{k}_{M r r}=0.240810^{-4} \mathrm{M} \mathrm{L} \mathrm{S}^{-3} \mathrm{~T}^{-1}$ \\
& $\mathbf{k}_{M r z}=\mathbf{k}_{M z r} \approx 0$ \\
& $\mathbf{k}_{M z z}=0.240510^{-4} \mathrm{M} \mathrm{L} \mathrm{S}^{-3} \mathrm{~T}^{-1}$ \\
& $H_{M r r}=0.1236 \mathrm{~L}^{3} \mathrm{~S} \mathrm{M}^{-1}$ \\
& $H_{M r z}=H_{M z r} \approx 0$ \\
& $H_{M z z}=0.1232 \mathrm{~L}^{3} \mathrm{~S} \mathrm{M}^{-1}$ \\
Cermeability tensor & $\mathbf{c}_{M r r}=0.1899 \mathrm{~L}^{2} \mathrm{~T}^{-2} \mathrm{~S}^{-1}$ \\
& $\mathbf{c}_{M r z}=\mathbf{c}_{M z r} \approx 0$ \\
& $\mathbf{c}_{M z z}=0.1868 \mathrm{~L}^{2} \mathrm{~T}^{-2} \mathrm{~S}^{-1}$ \\
Dissipation coupling tensor & $\mathbf{d}_{M r r}=0.0833 \mathrm{~L}^{3} \mathrm{M}^{-1} \mathrm{~T}^{-1} \mathrm{~S}$ \\
& $\mathbf{d}_{M r z}=\mathbf{d}_{M z r} \approx 0$ \\
& $\mathbf{d}_{M z z}=0.0819 \mathrm{~L}^{3} \mathrm{M}^{-1} \mathrm{~T}^{-1} \mathrm{~S}$ \\
\hline
\end{tabular}

Table 4: Homogenized material coefficients for case 5

\begin{tabular}{ccc}
\hline case & $\eta_{T}$ & $\eta_{F}$ \\
\hline 1 & 0.0968 & 0.0727 \\
2 & 0.0995 & 0.1017 \\
3 & 0.1058 & 0.1122 \\
4 & 0.1102 & 0.1171 \\
5 & 0.1145 & 0.1198 \\
\hline
\end{tabular}

Table 5: Evolution of fluid and thermic indicators

\begin{tabular}{cccccc}
\hline case & $n_{S}$ & $\max _{\Omega} \sigma$ & $\max _{\Omega} \sigma_{\text {ref }}$ & $\eta$ & $\eta_{\text {ref }}$ \\
\hline 1 & 2084 & 0.0233 & 0.0228 & 0.294 & 0.309 \\
2 & 4810 & 0.0246 & 0.0238 & 0.255 & 0.279 \\
3 & 7682 & 0.0255 & 0.0252 & 0.227 & 0.236 \\
4 & 10842 & 0.0303 & 0.0299 & 0.082 & 0.094 \\
5 & 15358 & 0.0314 & 0.0310 & 0.049 & 0.061 \\
6 & 26602 & & 0.0330 & & \\
\hline
\end{tabular}

Table 6: Evolution of maximal principal stresses / $0.7 \mathrm{GPa}$ and corresponding errors 

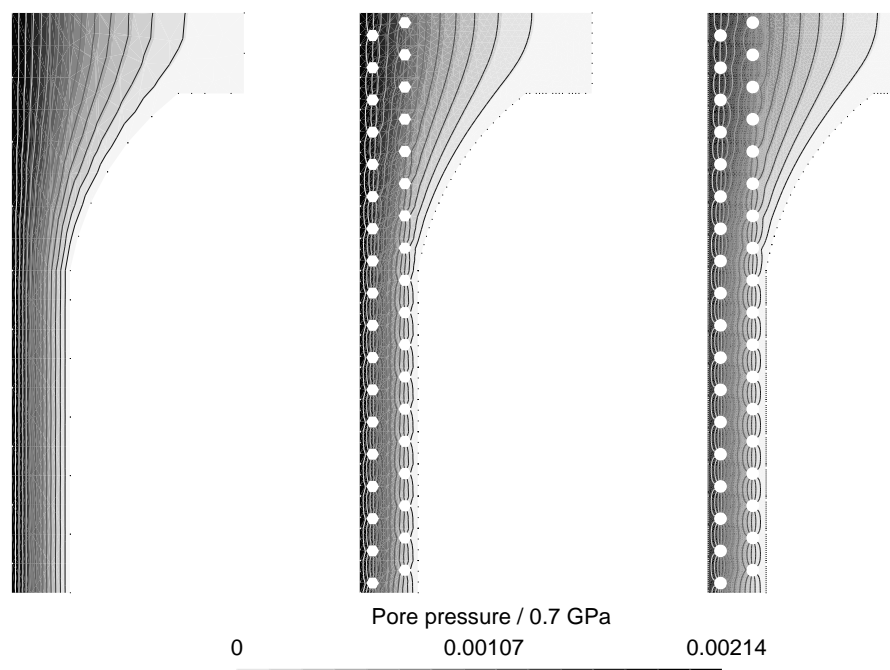

Figure 9: Pore pressure (case 1, left), reference pore pressure (case 1, middle) and target pore pressure (case 6 , right)
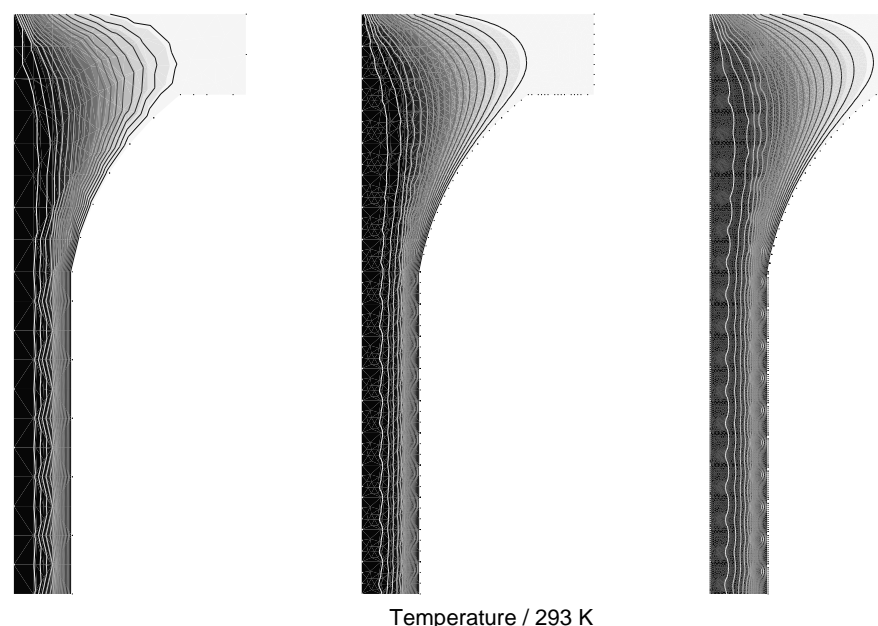

0

$$
0.137
$$

0.273

Figure 10: Temperature (case 1, left), reference temperature (case 1, middle) and target temperature (case 6 , right)

An adaptation to the classical periodic homogenization has been done for the case where only one direction of periodicity pertains. Edge effects in the 

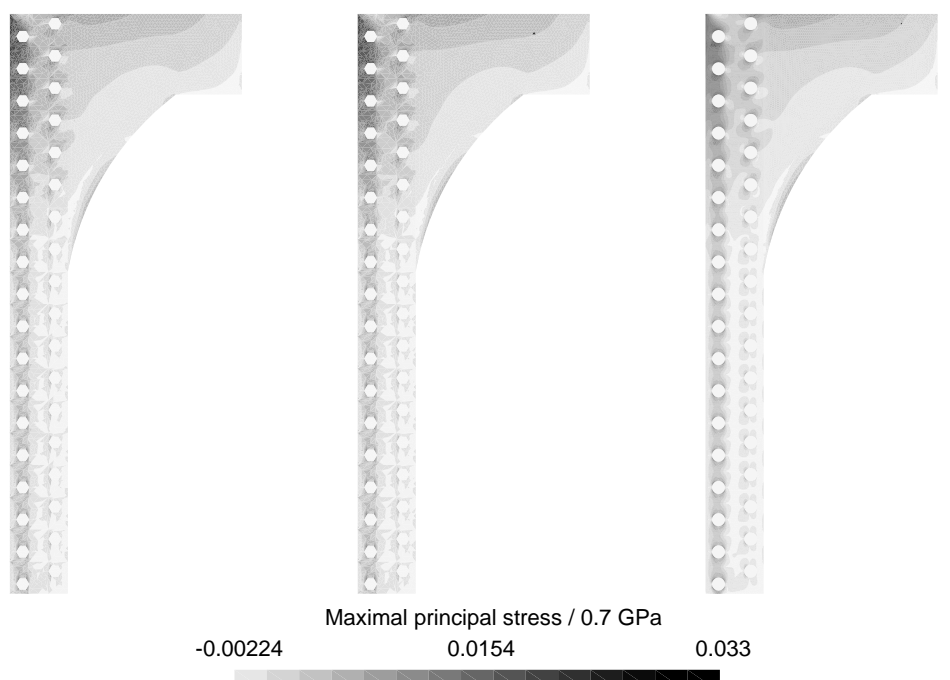

Figure 11: Maximal principal stress (case 1, left), reference field (case 1, middle) and target field (case 6 , right)

transverse directions are taken into account throughout the homogenized fields, with particular boundary conditions on the microscale cells, and thanks to the use of a projection between non conforming meshes.

The feasibility of this approach has been exemplified on an axisymmetric problem, and convergence was studied numerically when the mesh of the physics described up to the microscale is refined. Comparisons of different relocalization strategies are currently under development.

\section{References}

[1] O. Allix, E. Baranger, and L. Blanchard. An efficient strategy for the calculation of end effects on composite pipes: The thermoelastic case. Composite Structures, 76(4):291-302, 2006.

[2] E. Aulisa, S. Manservisi, and P. Seshaiyer. A non-conforming computational methodology for modeling coupled problems. Nonlinear Analysis, 63(5-7):1445-1454, 2005.

[3] F. B. Belgacem and Y. Maday. The mortar finite element method for three dimensional finite elements. Mathematical Modelling and Numerical Analysis, 31:289-302, 1997.

[4] A. Bensoussan, J.-L. Lions, and G. Papanicolaou. Asymptotic Analysis for Periodic Structures. North Holland, Amsterdam, 1978. 
[5] C. Bernardi, Y. Maday, and A. T. Patera. A new nonconforming approach to domain decomposition: The mortar element method. In H. Brezzi, editor, Nonlinear partial differential equations and their applications, pages 13-51. Paris, 1994.

[6] N. Buannic, P. Cartraud, and T. Quesnel. Homogenization of corrugated core sandwich panels. Composite Structures, 59:299-312, 2003.

[7] X.-C. Cai, M. Dryja, and M. Sarkis. Overlapping non-matching grid mortar element methods for elliptic problems. SIAM Journal on Numerical Analysis, 36:581-606, 1999.

[8] P. Causin, J.-F. Gerbeau, and F. Nobile. Added-mass effect in the design of partitioned algorithms for fluid-structure problems. Computer Methods in Applied Mechanics and Engineering, 194(42-44):4506-4527, 2005.

[9] R. Cools. An encyclopaedia of cubature formulas. Journal of Complexity, 19(3):445-453, 2003.

[10] O. Coussy. Poromechanics. John Wiley \& Sons, 2004.

[11] L. Dormieux, D. Kondo, and F.-J. Ulm. Microporomechanics. Wiley, 2006.

[12] D. Dureisseix and H. Bavestrello. Information transfer between incompatible finite element meshes: Application to coupled thermoviscoelasticity. Computer Methods in Applied Mechanics and Engineering, 195(44-47):6523-6541, 2006.

[13] D. Dureisseix, P. Ladevèze, and B. A. Schrefler. A LATIN computational strategy for multiphysics problems: Application to poroelasticity. International Journal for Numerical Methods in Engineering, 56(10):1489-1510, 2003.

[14] C. Farhat, M. Lesoinne, and P. LeTallec. Load and motion transfer algorithms for fluid/structure interaction problems with non-matching discrete interfaces: Momentum and energy conservation. optimal discretisation and application to aeroelasticity. Computer Methods in Applied Mechanics and Engineering, 157:95-114, 1998.

[15] C. A. Felippa, K. C. Park, and C. Farhat. Partitioned analysis of coupled mechanical systems. Computer Methods in Applied Mechanics and Engineering, 190:3247-3270, 2001.

[16] J. Fish and V. Belsky. Multigrid method for periodic heterogeneous media. Part 1. Computer Methods in Applied Mechanics and Engineering, 126:1$16,1995$.

[17] P.-A. Guidault, O. Allix, and J.-P. Navarro. A two-scale approach with homogenization for the computation of cracked structures. Computers and Structures, 85(17-18):1360-1371, 2007. 
[18] P. Hillion. Numerical integration on a triangle. International Journal For Numerical Methods In Engineering, 11(5):797-815, 1977.

[19] J. Hohe and W. Becker. Effective stress?strain relations for twodimensional cellular sandwich cores: Homogenization, material models, and properties. Applied Mechanical Review, 55(1):61-87, 2002.

[20] P. Ladevèze and D. Dureisseix. Une nouvelle stratégie de calcul micro/macro en mécanique des structures. Comptes-Rendus de l'Académie des Sciences, 327(12):1237-1244, 1999.

[21] P. Ladevèze, O. Loiseau, and D. Dureisseix. A micro-macro and parallel computational strategy for highly heterogeneous structures. International Journal for Numerical Methods in Engineering, 52(1-2):121-138, 2001.

[22] R. W. Lewis and B. A. Schrefler. The Finite Element Method in the Static and Dynamic Deformation and Consolidation of Porous Media. John Wiley \& Sons, 2nd edition, 1998.

[23] R. Matteazzi, B. A. Schrefler, and R. Vitaliani. Comparisons of partitioned solution procedures for transient coupled problems in sequential and parallel processing, pages 351-357. Advances in Computational Structures Technology. Civil-Comp Ltd, 1996.

[24] D. Néron and D. Dureisseix. A computational strategy for poroelastic problems with a time interface between coupled physics. International Journal for Numerical Methods in Engineering, 2007. To appear.

[25] D. Néron and D. Dureisseix. A computational strategy for thermoporoelastic structures with a time-space interface coupling. International Journal for Numerical Methods in Engineering, 2007. To appear.

[26] J.-F. Remacle, N. Chevaugeon, E. Marchandise, and C. Geuzaine. Efficient visualization of high-order finite elements. International Journal For Numerical Methods In Engineering, 69(4):750-771, 2007.

[27] E. Sanchez-Palencia. Non Homogeneous Media and Vibration Theory, volume 127 of Lecture Notes in Physics. Springer Verlag, 1980.

[28] T. Zohdi, J. T. Oden, and G. J. Rodin. Hierarchical modelling of heterogeneous bodies. Computer Methods in Applied Mechanics and Engineering, 138:273-298, 1996. 